\title{
Social and Personal Disclosure in a Location-Based Real Time Dating App
}

\author{
Colin Fitzpatrick \\ Northwestern University \\ fitzcn@u.northwestern.edu
}

\author{
Jeremy Birnholtz \\ Northwestern University \\ jeremyb@northwestern.edu
}

\author{
Jed R. Brubaker \\ University of California - Irvine \\ jed.brubaker@uci.edu
}

\begin{abstract}
Users of location-based real time dating (LBRTD) apps must decide what to disclose in their profiles. Based on 25,365 profiles from one app, our study explores users whose profile photos disclose a face, a personal disclosure that identifies them as an individual, in relation to available social disclosures, those that do not identify them as an individual. Using logistic regression models with face disclosure as the outcome, we find: older users, and users who share race are less likely to disclose a face, and higher body mass index (BMI) users, users who disclose relationship status, and users who are seeking friends or relationships are more likely to disclose a face. We frame and discuss these results in relation to literature on location norms, body image, race relations, and social stigma, and propose future work to investigate these findings, particularly those of race, relationship status, and goals, in qualitative depth.
\end{abstract}

\section{Introduction}

People increasingly turn towards technology to facilitate encounters with others, from the friendly to the sexual, to the romantic [32]. Grindr, a locationbased real time "dating" (LBRTD) app designed for men who have sex with men (MSM), is one app that supports all three of these types of interactions [37]. Grindr affords social exploration by supporting profile creation and providing logged-on users access to profiles of and the ability to chat with logged-on, nearby others. Grindr's architecture is unique because it is not a stable network but rather an ephemeral mutual visibility based on time and distance.

As is common with other dating and networking sites (e.g., [7]), Grindr profiles are a reduced set of identifying cues compared with face-to-face spaces. As we begin to understand how these apps are used, one important question concerns the information users choose to disclose in their profiles. The opportunities for disclosure in a Grindr profile include: one photo, distance, age, height, weight, race, relationship status, and goal (a multiple select category of networking, chat, friends, dates, relationship, and fun). Profiles are typically the first point of contact between users and therefore impact impression formation and how users discern the attractiveness and motives of others [4,5].

However, it is unlikely that all types of profile information play an equal role. Textual or numeric information, such as height, weight, age, and relationship status, for example, conveys general information that can contribute to impression formation, but is unlikely to render an individual identifiable or provide a full picture of a users' appearance or attractiveness.

Photographs of a user's face, on the other hand, provide individuating detail that could convey attractiveness, but might render the user identifiable by others in unwanted ways [4]. Photos may also deliberately or inadvertently - convey information about race or body type, which have been the subject of some controversy on apps like Grindr [1,19,33].

While it may seem initially like people would want to maximize their attractiveness by disclosing more information, more careful consideration suggests otherwise. Someone seeking casual sex partners while claiming to be "in a relationship," for example, may be less willing to disclose information than a "single" user with the same goal. Still others may not feel confident enough to share a photo of their face.

By examining patterns of disclosure in profiles on Grindr, the aim of this paper is to better understand what users share identifying information, faces, and what factors seem to influence this sharing behavior. To do so, we explore four key questions on face photo sharing related to sharing of physical traits, relationship status, race, and goals on the app. We ground this exploration by drawing on literature in the areas of self-categorization and personal and social attraction, studies of MSM and sharing in reduced-cues environments, and race relations within MSM communities.

\section{Background literature}

The focus of this paper is an exploration of the combination of different types of disclosure available 
in a given Grindr profile, social (age, height, weight, relationship status, race, and goals) and personal (face photos). These profile fields are the tools that individuals have for self-presentation, and their sharing of them represent a negotiation of disclosure to their desired or "imagined audience" [25] within the app, their conceptualization of who they are constructing their profile for. While this paper does not assume to know how other users interpret these disclosures, it does argue that critical relationships between social and personal disclosures exist. Literature on social and personal attraction offers a theoretical foundation for exploring our research questions, and consideration of each is supplemented by topical literature.

\subsection{Social and personal attraction}

Several theories address identity and disclosure in reduced-cues CMC environments, of which research on self-categorization is most directly relevant here. Building on early social identity theory by Tajfel and Turner [35], Turner's [36] self-categorization theory positions identity as simultaneous combinations of superordinate (most abstract, such as "human"), intermediate (a group, such as "married people"), and subordinate categories (an individual, "John Doe"). Self-categorization theory later led to the social identification/deindividuation (SIDE) model to study groups and individuals in CMC contexts [22,34].

Researchers have also applied and adopted these theories in studying the facilitation of romantic/casual encounters in CMC environments. Lea and Spears [23] discuss social and personal attraction in studying disclosures of users in $\mathrm{CMC}$, connecting social attraction to social categorization and personal attraction to individuation. Jones, in his study of a Hong Kong chat room for MSM, applies social and personal attraction to discuss the tensions users face in negotiating the shift from text-based communication (broadly social attraction) to, eventually, known face images or video (personal attraction) [18].

Faces represent a personal disclosure because of their biological uniqueness and the ways in which viewers process them. Researchers in biometrics have found that faces can be used for identification purposes [17], and that they may be used in combination with other biometrics for increased accuracy in identifying individuals [15]. From a human perception perspective, Peterson discusses the ways in which people process faces holistically and how people may use their facial processing to differentiate between people [29]. In this way, faces on Grindr become identifying than disclosure via profile fields such as age, relationship status, or particular goals.
Gross and Acquisti have shown that identifiability of profile photos differs depending on the social network site in use [11]. When considering the role pictures play in profiles for romantic encounters, Hancock et al. found that a third of photos of heterosexual users of an online dating site had some sort of discrepancy when evaluated by independent judges [14]. It is important to note that the schema for [14]'s study included questions on hair and teeth, suggesting the photos studied regularly included faces. Earlier studies of MSM populations discuss dating profile photos as containing faces [5], but more recent work shows that on LBRTDs, some users do not include a face photo for various reasons including privacy and managing the risk of being identified within a potentially stigmatized environment [4].

Faces, then, maintain a particular significance within identity literature around personal and social attraction and within contextual literature about LBRTDs and Grindr in particular. We explore our data to capture emergent patterns around face disclosure while considering effects of location, selfcategorizations, and goals of users. Patterns shape the discussion around the practices of social and personal disclosure in LBRTDs and help inform more nuanced questions for future work.

\subsection{Location}

Another key issue is that of location. Perhaps Grindr's most defining characteristic is that it shows a user the other fifty users closest to them who are either online or have been online in the past hour. Mowlabocus theorizes that the ephemerality of Grindr's articulated visibility across users, dependent on space and time, makes it impossible to ever "go into" a particular network or space on the app [28]. Still, as Blackwell et al. show, Grindr creates areas of co-situation among users who log into the same Grindr space but are located in disparate physical contexts [4]. Relatedly, Humphreys argued in a study of Dodgeball that location-based social networks in urban public spaces influence norms around interaction with other users [16]; Licoppe et al. found that social norms around interaction with spaces and locations emerged on a different location-based game service [24].

Moreover, these and other studies show that users of location-based services confront privacy concerns, enhanced through location data. Licoppe et al. found that players of the game had to manage disclosure of location and potential danger of stalkers. More broadly speaking, Barkhuus et al., in a general study around apps and privacy concerns, found that users had a much higher level on concern for privacy for apps that used location data [3]. Given the combination of 
location-based services affecting social interaction with other users, with particular places and locations, and privacy concerns, we ask:

RQ1. Are there geographically local norms around individuating disclosures of face on Grindr?

\subsection{Physical characteristics}

In text-based communication in pursuit of romantic or sexual encounters (in person or online), it is common for users to include basic stats describing themselves early in their exchange. Gudelunas found, in his study of gay and lesbian online personal ads, that many ads by men included details about their age, height and weight [12]. In the more synchronously interactive medium of text-based chat, Jones also highlights users' practice of negotiating the disclosure of age, height and weight near the beginning of their interactions [18]. As Jones shows in chat rooms, these three pieces of information are disclosed at the same time and thought of as a unit [18]; in our following analyses we will look at them together as a group.

The combination of these stats may influence the way a user views himself or the ways in which others view him. There is an ongoing conversation in popular press and opinion pieces about body image of homosexuals. The conversation centers around the increasing amount of homosexual men who suffer from body image disorders [21] and how these issues are played out on image consciousness social platforms like Grindr and Facebook [20]. For photos on Grindr, this emerges most clearly through what is referred to as the headless torso profile $[4,19]$. We therefore ask:

RQ2. How likely are users to individuate themselves through a face photo based on physical characteristics of age and body mass index?

\subsection{Social categorizations}

In considering how people disclose information about themselves, there is evidence in prior work to suggest that social categorizations are important. In research on dating sites, Ellison et al. found that users balance tensions between being honest and portraying an idealized self when reporting on parts of their dating profiles, such as athleticism, interest in sex, and other social categories [8]. In a later study, Ellison et al. found that reduced cues, asynchronicity, and context factored into profile discrepancies [9].

There are two profile categories that may become influential in disclosure for Grindr users: race and relationships status. Han's treatment of race in the gay community details arguments about the ways nonWhite racial identities are rendered invisible through prioritization of the white body in these spaces, evidenced in equality efforts selling "gay" as "white" and "familiar" [13]. Building on Fanon's arguments [10] around sexualization and fetishization of black bodies in MSM communities, Han argues that queer men of color lack agency and individuality.

Opinion pieces on Grindr and race, such as $[1,19,33]$, discuss the privileging of the white male body and denying of others through explicit profile statements regarding attraction (or lack of attraction) based on race like "white guys to the front, no Asians, no blacks;" this racialization of attraction by some users on Grindr might suggest that face photo disclosure would also be different. We therefore ask:

$R Q 3 a:$ Is there a relationship between disclosure of particular races and disclosure of a face photo?

In a reduced cue environment such as Grindr, relationship status is mutable. Because Grindr's design supports multiple relationship status types (including "single," "married," and "open relationship"), disclosures of certain types may be predictive of disclosure of a face as well; alternatively, those who do not disclose a relationship status may not disclose a face to remain more anonymous. Given this mutability of relationship status, we explore:

RQ3b: Is there a relationship between disclosure of particular relationship statuses and face disclosures?

\subsection{Goals}

Disclosure also may depend on context and goals. As Gross and Acquisti show, revelation practices are different across different social network sites, each with an arguably distinct goal in mind [11]. Grindr supports several different goals, each of which may be explicitly identified in the profile: chat, friends, dates, relationship, networking, and, at the time we collected data, fun. As a multiple-select category, users can express multiple goals at the same time, and therefore collapse the context for revelation disclosure that Gross and Acquisti find [11]. Bumgarner's ethnographic treatment of Grindr explores these different types of goals and find that the app indeed supports more interaction than just hookups [6]; in a later research study, Blackwell et al. expand on the importance of these different goal types and co-situation when considering how Grindr users present themselves to others [4]. We asked:

RQ4. Do any particular goals impact the likelihood a user will disclose a face?

\section{Methods}


Data was collected from twelve different locations ( six cities and six college towns) across the United States and Canada between May 2011 and February 2012. Data collection started with select cities and more were added throughout the process; no data in this set was collected from October $10^{\text {th }}$ through November 15. The specific coordinates used for each location did not change during data collection. We have no reason to believe that, other than different sample sizes, the collections at different time periods would affect disclosure behavior.

Collection was performed using a custom written Java application. Simulating a user, the application "logged on" in the twelve locations every ten minutes and collected profile data of each of the fifty profiles visible. In total, the data includes 53,426 observations of 38,402 unique users. We prepped the data for our analyses, which focuses on the first appearance of a unique users in twelve selected locations, by dropping observations from users outside our twelve locations $(n=18220)$, observations that were incomplete from changes in the apps service or database storage issues $(n=161)$, and second or later observations of a user to end with 25,365 first observations of users in our twelve locations.

Following the work of Bakhshi et al. ([2]) we ran the collected profile photos through the Face++ face detection API [27]. We validated Face++ performance through human coding for presence or absence of a face in a random sample of 1,000 of our 25,365 photos. A member of the research team hand coded the photos for an identifiable face (roughly $2 / 3$ or more of the face was visible and unobstructed) or an unidentifiable face/no face. We found $86.4 \%$ agreement between the human coding and the API detection.

\begin{tabular}{lc}
\hline \multicolumn{2}{c}{ Table 1. Human coded face data $(\mathrm{n}=1000)$} \\
\hline Face & $68.8 \%$ \\
Headless torso & $17.6 \%$ \\
Shirt & $15.3 \%$ \\
No Shirt & $84.7 \%$ \\
Human (no face, no torso) & $3.5 \%$ \\
Non-Human & $10.1 \%$ \\
Under-review & $25.7 \%$ \\
Monochrome (blank) & $24.7 \%$ \\
Object & $49.6 \%$ \\
\hline Results of human coding of photos and \\
subcategories. Categories are mutually \\
exclusive so "human" photos do not include \\
faces or torsos (i.e. legs, feet, arm, etc.).
\end{tabular}

We decided to hand code further to see the proportions of the types of photos that Grindr users have. For the 1,000 photos that we compared our results to the API, we also coded for torso/no torso, shirt/no shirt, and human/non-human. The results, reported in Table 1, show a spread of the face/no-face categorization we are looking at for this paper. Though it would be a worthwhile inquiry to look at the torso and the shirtless torso profiles in particular, as we do not consider them individuating disclosures, we will not further analyze them here.

Given the strong results from our hand-coding validation of the Face++ API, we used the API results for subsequent analyses. With a binary outcome variable (face, no face), and both continuous (age, height, weight) and categorical (location, ethnicity, relationship status, and goals) variables, we used logistic regression models to explore $R Q 1-R Q 4$ around combinations of disclosure practices of users. In total we ran four models, adding more variables to each model. We started with locations in M1, then added physical characteristics in M2, social categories in M3, and finally goals in M4.

\section{Results}

\subsection{Descriptive statistics}

We initially explored our data by running a series of descriptive statistics. We share these in Table 2 to familiarize readers with our dataset. It should be made clear that not all of these data are structured in the same way. Location was recorded by the Java application at collection time, and so does not represent a profile field or a disclosure from the user. Sharing distance is a single binary option in the profile, while relationship status and ethnicity are multiple choice, single response categories with "do not share" as the default response when creating a profile. The goal data comes from a multiple choice, multiple response category in the profile; each goal was treated separately as a dummy variable in analysis to control for the 27 different combinations of the goals in the profiles used in the analyses.

\begin{tabular}{llr}
\hline $\begin{array}{c}\text { Table 2. Descriptive statistics of variables } \\
\text { in models (N=25,365, unless noted) }\end{array}$ \\
\hline \multicolumn{2}{c}{ Categorical Variables } \\
\hline Face detected & \multicolumn{2}{c}{$54.26 \%$} \\
Location & City A & $19.09 \%$ \\
& College A & $10.55 \%$ \\
& College B & $11.70 \%$ \\
& College C & $11.04 \%$ \\
& City B & $15.55 \%$ \\
& City C & $.44 \%$ \\
& City D & $12.56 \%$ \\
& College D & $14.97 \%$ \\
& City E & $2.57 \%$ \\
& City F & $.57 \%$ \\
& College E & $.45 \%$ \\
& College F & $.50 \%$ \\
Share Distance & & $86.86 \%$
\end{tabular}




\begin{tabular}{|c|c|c|}
\hline \multicolumn{3}{|l|}{ Relationship } \\
\hline & Do not share & $47.63 \%$ \\
\hline & Single & $44.36 \%$ \\
\hline & Dating & $1.10 \%$ \\
\hline & Exclusive & $.38 \%$ \\
\hline & Committed & $.76 \%$ \\
\hline & Partnered & $3.21 \%$ \\
\hline & Engaged & $.24 \%$ \\
\hline & Married & $.59 \%$ \\
\hline & Open & $1.73 \%$ \\
\hline \multicolumn{3}{|l|}{ Ethnicity } \\
\hline & Do not share & $25.24 \%$ \\
\hline & Asian & $5.30 \%$ \\
\hline & Black & $3.15 \%$ \\
\hline & Latino & $8.67 \%$ \\
\hline & Middle Eastern & $.84 \%$ \\
\hline & Mixed & $5.77 \%$ \\
\hline & Native American & $.18 \%$ \\
\hline & White & $49.31 \%$ \\
\hline & South Asian & $1.31 \%$ \\
\hline & Other & $.24 \%$ \\
\hline \multicolumn{3}{|l|}{ Goals* } \\
\hline & Fun & $2.96 \%$ \\
\hline & Chat & $36.75 \%$ \\
\hline & Friends & $43.40 \%$ \\
\hline & Relationship & $20.95 \%$ \\
\hline & Dates & $31.74 \%$ \\
\hline & Networking & $23.34 \%$ \\
\hline & \multicolumn{2}{|c|}{ Continuous Variables** } \\
\hline \multirow{3}{*}{$\begin{array}{l}\text { Age in years } \\
(\mathrm{N}=20085) \\
\text { BMI } \\
(\mathrm{N}=18380)\end{array}$} & Mean & SD \\
\hline & 29.97 & 8.58 \\
\hline & 23.94 & 3.61 \\
\hline \multicolumn{3}{|c|}{$\begin{array}{l}{ }^{*} \text { Goals, a multiple response category, will } \\
\text { add up to more than } 100 \% \text {. } \\
\text { }{ }^{\star *} \text { These variables rely on users disclosing } \\
\text { that information, and so have smaller N's. }\end{array}$} \\
\hline
\end{tabular}

\subsection{Logistic regression models}

The results from all four models are reported in Table 3. Here we discuss each model in light of the research questions outlined above.

4.2.1. Locations (RQ1). We ran M1 to answer $R Q 1$, which asked whether locations had a significant effect on face disclosures. While it might be the case that other features determine whether or not someone discloses a face, it could be that there are localized norms around face disclosure that are affected by location and other users at that. M1 shows that four of the twelve locations emerge as significant, two cities and two college towns; this baseline of significance for these locations will be tracked in later models to see if locations' significance remains when adding other variables. Sharing distance from other users was also included in the model. While Grindr does not, on profiles, support users giving their exact location, it does support distance sharing. Zhao et al. have found that location disclosure in location-based social networks (LBSNs) correlates with personalization [39].
We used Grindr's share distance to capture, as best as possible, a similar effect.

It is important to note that because of collinearity in the model (as each user has one and only one location), City A is included as the reference category that the other locations are being compared to for significance. Using the largest categorical value as the reference category is common practice in logistic regressions [26]. The odds ratios in the table can be interpreted as change in odds between City A and other locations: for example, the odds of a user in location "City E" disclosing a face are 1.42 times higher than those of someone in reference category "City A," when controlling for sharing distance.

Though M1 shows four locations in the model to be significant, not all locations are and the model does not account for several other variables. It could be that as other variables are added to later models, the significance of the four locations could change and/or the direction of the effects could change so that users would be less likely to disclose a face as compared to users in "City A."

4.2.2. Physical characteristics (RQ2). Model 2 introduces the disclosure of physical information, captured in the age and Body Mass Index (BMI) (where $B M I=\frac{\text { weight }(l b)}{\text { height }(\text { in })^{2}} * 703$, following [38]); because this calculation requires revelation of this information in the profile, the overall $\mathrm{N}$ in $\mathrm{M} 2$ and subsequent models $(\mathrm{N}=15,897)$ is lower than that of M1 $(\mathrm{N}=25,365)$. Overall, $79.2 \%$ of users in the set for analysis reveal age and 72.4 reveal height and weight to be able to compute BMI. Our interest in the effect of the change in these variables, not just their disclosure, warrants the lower N. Given literature on homosexuality and body image reported above, it would make sense that those who are younger and with a lower BMI, and thus fitting a desired body image, would be more likely to disclose a face than arguably less desirable others who are older and/or heavier.

Both age and BMI are found to be significant within Model 2, though the odds ratio is close to one, which means that only large changes would lead to meaningful changes in the probability of a face disclosure. These odds ratios are predictive in opposite directions, however. For each increase in age of one year, the odds of a user disclosing a face decreases by a factor of .99 , controlling for other variables in the model; for each one unit increase in BMI, the odds of a user disclosing a face increases by a factor of 1.01, controlling for other variables. Predicting marginal effects offers a different way to think about this same finding. Examples of extreme cases help illustrate the difference: Person A, a younger man (21 years old) with a higher BMI (30), has a 58.96\% likelihood of 
disclosing a face whereas Person B, an older man (45) with a lower BMI (20), has a $49.63 \%$ chance of disclosing a face when using Model 2.

M2 shows a surprising finding with regard to physical characteristics. While it would stand to reason that younger users and those with lower BMIs might be more likely to disclose a face, the model shows the opposite for BMI. It could be that users with lower BMIs are more likely to have torso profiles, to show off a sculpted body, and/or users with a higher BMI obscure or crop out their torso in order to keep a visual representation of their bodies away from other users. The former, which gets at profile disclosures, could be addressed with additional modeling that includes torso data that we did not pursue given the $\mathrm{N}$ of the data and absence of a torso detection algorithm; the latter, which addresses a question about user decisions, cannot be answered conclusively with this data.

4.2.3. Social-categorizations (RQ3a and RQ3b). We next added the social-categorization variables in M3 to answer $R Q 3 a$ and $R Q 3 b$ concerning effects of racial and relationship status disclosures on face disclosure. Given literature and opinion pieces on race and visibility within the gay community, it may be that users who identify as non-white include their faces in order to be seen; on the other hand, however, it might be that disclosing a non-white race effects individuating behaviors in some way and those users are less likely to have a face.

For relationship status, given the original use of Grindr for a dating and/or hookup app, one may assume that users who are in a relationship, committed, engaged, partnered, or married would be less likely to show a face and become a known individual given that they are already in a relationship with someone else; relatedly, an user who is single may be actively trying to individuate himself using his face to attract others.

The reference categories for both of these variables are the "Do Not Disclose" category. This reference category is unique in that it is the default value of a profile and a user does not need to make an active choice for it. That said, it is useful when studying the effects of disclosing social categories to keep those who do not disclose in the model in some way. Therefore, in M3 and M4 for the ethnicity and relationship status variables, the odds ratios are being reported in terms of someone who did not disclose within the category, rather than another nominal, chosen value within that category.

For the race category, disclosing "Black" or "South Asian" are both significant in predicting a decrease in likelihood to disclose a face. For the relationship category, disclosures of "Single," "Dating," "Committed," "Partnered," and "Open" all predict an increase in likelihood of disclosing a face.
Variables that were significant in M1 and M2 remain significant in the same direction and their odds ratios are similar. (The possibility of racial bias in the face detection API is discussed in the limitations section.)

4.2.4. Goals (RQ4). $R Q 4$, our final question, asked whether or not goals are significant in predicting face disclosures on Grindr. Given the broad range of goals available to Grindr users, it may be the case that users who select particular goals are more or less likely to disclose a face. It would make sense that those who are looking for dating or relationship might be more likely to have a face in their photos than those who are looking for fun, chat, or friends (especially if users view those categories as euphemisms).

M4 offers the most complete look at predicting probabilities of a user making a face disclosure by adding goals, coded as dummy variables, in the model. Only two of the goals, "Friends" and "Relationship" are significant, with odds ratios of 1.12 and 1.14 , respectively, meaning that a disclosure of either would increase the likelihood of disclosure a face by a factor of 1.12 or 1.14 , controlling for other variables present in Model 4. Again, the significant variables from the earlier models remain significant in Model 4. For race, "Black" and "South Asian" remain significant in the same direction and around a similar odds ratio. The relationship categories that were significant remain so, still predicting a positive increase in likelihood, but each with a slight decrease in their odds ratio. Age, BMI, sharing distance, and each of the four significant locations all also remain significant in the same direction and with the similar odds ratios as before. It is important to note that the "White" category for racial disclosure has become significant in Model 4 where it previously was not. While like the two other race disclosures in that it predicts a decrease in the likelihood of a face disclosure, its odds ratio is much closer to one (.91 instead of .65 or .70 for "Black" and "South Asian" respectively).

Marginal predictions for M4 may help think about how the odds ratios and disclosure come together for thinking about the likelihood of a given user disclosing a face. An example of an extreme unlikely case of disclosing a face would be a user in City F, not sharing distance, older (45), lower BMI (20), married, Black, and looking for chat; he would have a 14\% likelihood of disclosing a face. An example of an extreme likely case would be a younger (21), higher BMI (30) user in "College F" who shares distances, does not share ethnicity, is single, and is looking for fun, friends, relationship, dates, and networking; he would have a $82.78 \%$ likelihood of disclosing a face in his profile picture. Based on the descriptive statistics, the predicted likelihood of an "average" user (defined by majority categories and means of City A, sharing 


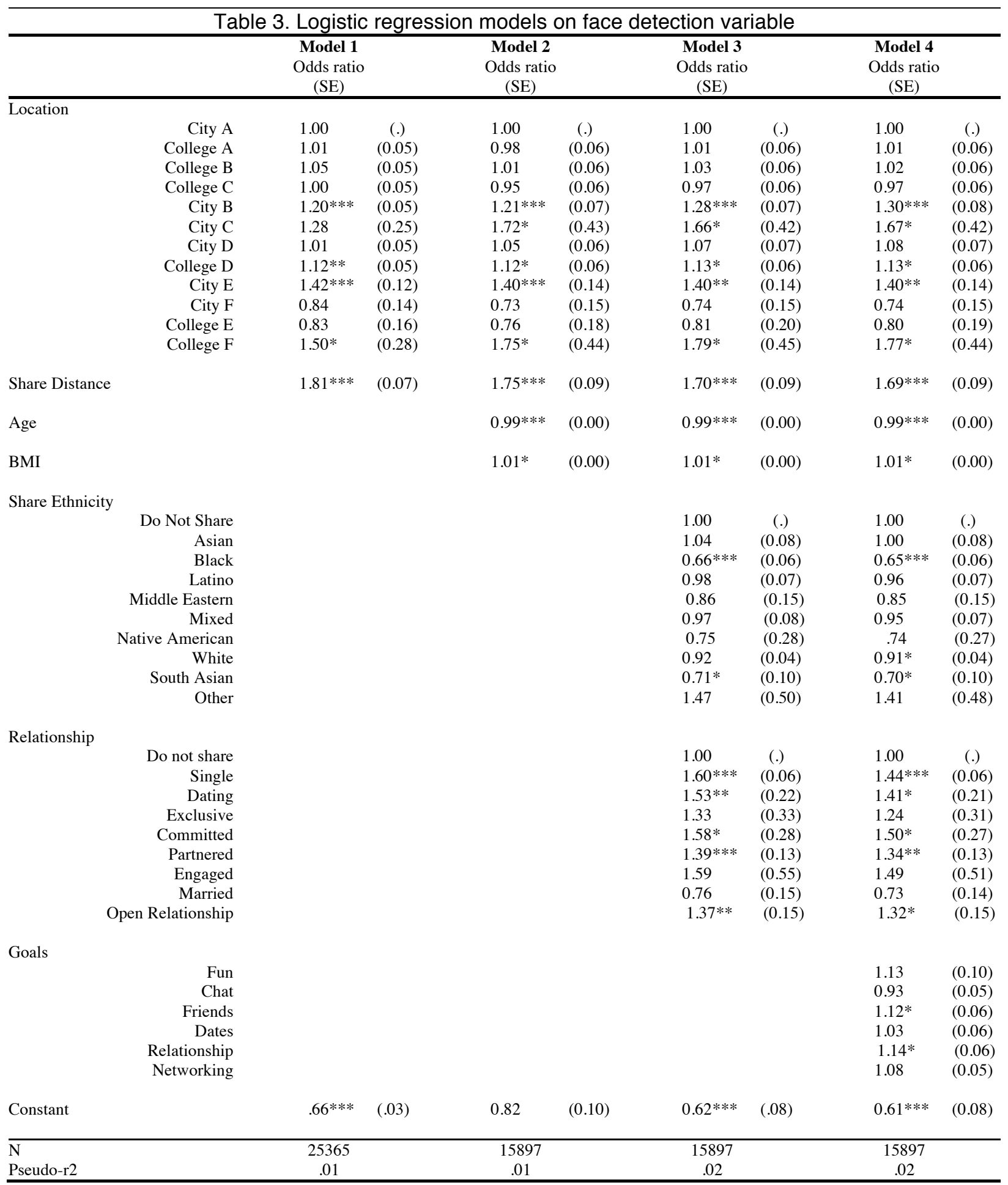

distance, 30 years old, 24 BMI, not disclosing relationship status, white, and looking for friends) disclosing a face is $50.9 \%$.

4.2.4. Looking across models. M1 was used to set a baseline for looking at significance of location on disclosure practices, given that context matters a lot for Grindr users. As later models introduce physical traits, self-categorizations, and goals, one might expect to see a depression of the effects of significant locations from M1. The locations, however, remained significant 
across all four models, suggesting that location has a robust effect for predicting face disclosures. Notably, odds ratios for some of the cities actually increased in the later models.

The age and BMI variables also remained significant across the models, though the more complete model changes the outcome of their effects. Looking at the marginal effects for predicting probability of face disclosure for the conditions of Person A and Person B previously computed for M2 shows that in the more robust M4, Person A's likelihood is $59.47 \%$ and B's is $52.16 \%$. This change demonstrates the influence in the effects of controlling for the other variables in the model.

\section{Discussion}

Looking at these results more generally, several considerations emerge. The findings show that the probability of individuating oneself within Grindr depends on a combination of the social disclosures available, from the local context, one's own body stats, race, relationship status, and goals.

\subsection{Geographic context matters}

Even after controlling for the other variables in later models, the four locations remained significant. This suggests that either local norms tied to geography, to the visible cascade of Grindr users on top of the geographic, or some combination of the both affect the amount to which people individualize themselves on Grindr with face photos. Given the various layers of locations, political climate, LGTB equality issues, positions towards casual sex, this could mean that users in particular locations do not face as much stigma in being on the app as others might. Further considering [24]'s finding around disclosure and social interaction in a LBSN, it may also be that certain geographic contexts support a sociality among individuals in the app in a way that other locations do not.

\subsection{Put best face (or torso) forward}

Disclosure around the body worked in opposite directions as age and BMI increased, with older users and users with a lower BMI being less likely to show a face. In an environment where users are trying to attract others, this finding shows a tenable argument around putting "best" physical features forward. If someone has a higher BMI, in photos he may be more likely to appear as large and, potentially, unattractive to other Grindr users; speculating, this may mean he crops his photo tighter on his face than his lower BMI counterparts. In the other case, the user with a lower BMI may be more likely to be a headless torso, to show off the rest of their body, a valued feature.

Age may work in a similar way, in that faces may give away more about age than other features of the body; with younger individuals potentially being prioritized, older users may strategize their interactions by not including a face. Alternatively, older users may also be more established within a particular geographic location and therefore more susceptible to negative impacts on reputation if found to be an individual user on the app - it would be better to first present at an abstraction and the negotiate an individuation disclosure after chatting with a particular other user (this idea, in part, comes from [4]). We plan to explore these questions further in another study using the handcoded data that breaks photos into more categories.

\subsection{Self-categorizations work differently}

In our exploration of two self-categorization disclosures, we found that the type of disclosure, race and relationship status, affect the probability of face disclosure in opposite directions. All the significant race self-categorizations decreased and all the relationship self-categorizations increased the probability of disclosing a face.

For race, it could be that a user who includes a face photo place conclusions about race in the eyes of the viewer of his photo. Race, though perhaps not mutable, may become ambiguous or subject to viewers' assumptions given a face photo. For race disclosures that do not include a face, then, users may disclose race in order to inform viewers given the reduction of other cues (faces).

Relationship status is curious in that both users who are single and not single (in various categories) are all more likely to disclose a face. It could simply be that those who are willing to disclose something that would not be given off by a photo (i.e. whether they are single, dating, married, etc.) would be more likely to have that photo be individuating because they might share more in general. Further qualitative examination for reasons of face disclosure across relationship status might uncover reasons behind this.

\subsection{Relationship goals predict personal disclosure}

While disclosing relationship statuses of various types increase probability of a face disclosure, only two goal types, "friends" and "relationship," are significant, and both increase the probability. The goal types may be grouped together in those that are potentially fleeting, 
and non-committed (fun, networking, dating, chat), and those that suggest something lasting (friends, relationship). For fleeting goals, disclosing a face may be less important; for goals that involve more lasting relationships, it may be important to individualize oneself from the beginning with a face photo.

\subsection{Limitations}

Our analyses are not without limitations that urge interpretation with caution. They represent only the first appearance of a set of users from particular places and at particular times and therefore do not account for changes in profile photos that might change face disclosures. Given potential for changes in disclosure norms from an increase or decrease in the number of users within a particular location and/or the changing context of MSM life within that location, these results may not represent current disclosure practices on the app. We have no reason to believe that these crosssections would systematically bias the general trends of face disclosure, even if it does not account for longitudinal change, because the significance of location does not account for all the significant variables in the models (which show significant userlevel variables that would not change in the same way).

Additionally, these results are only from Grindr, one of several LBRTD apps available, and an app for MSM; face disclosure practices may differ across these apps and may differ when users are seeking interactions with the opposite sex. Though this limits the generalizability of our findings to other LBRTDs and to LBSN systems at large, our results provide a footing for investigation of similar topics across the app and network landscape.

Finally, for findings on race in particular, there may be an instrumental bias for particular races in the Face++ algorithm. Phillips and O'Toole outline the challenges of face detection and recognition algorithms [30], and report findings from a study that demonstrates an other-race effect, where there is a bias of algorithms to detect faces of races of people that the algorithm was trained on [31]. Without validation on an external dataset of known racial identities of faces, interpreting the Face++ detection results must consideration potential for racial bias. While we have a hand-coded validation of face detection for 1,000 representative photos, we are unable to test this bias on that subset without assuming racial identities of users.

\section{Conclusions and future work}

We began by theoretically framing the different types of disclosures Grindr supports into two broad types, social and personal, and arguing that disclosures within profile fields were social and disclosures of a face photo were personal. With that framing in mind, we asked four questions around the patterns of disclosure to explore how social and personal disclosures interact and how location may influence personal disclosure. We found that various types of social disclosure and locations can predict the probabilities of personal disclosure in a LBRTD, but that some social disclosure reduce the likelihood and others increase the likelihood.

We plan to examine the findings presented in this paper with future studies, with further analyses of this data and collection of new qualitative data to help answer open questions. With qualitative data, we hope to gain an understanding about users' decision-making process around the things they share in the profile and the types of interactions that they have with other users based on their profiles. We further hope to look more closely at race, given not only the strong attention of opinion pieces on Grindr, but also the effects presented here, and the potential for the API to be biased.

These findings broadly speak to research on user practices and norms in location-based mobile services. By understanding how users interact with these types of systems, and how much they are willing to share with others concerning their identifiability, we will be better able to design for a broadening landscape of applications that enable contextual interactions with users based on location and time.

\section{References}

[1] Artrip, N. No Fat, No Femme. thefeministwire.com, 2013. http://thefeministwire.com/2013/11/no-fat-no-femme-thepolitics-of-grindr/.[2] Bakhshi, S., Shamma, D.A., and Gilbert, E. Faces engage us. ACM Press, 2014, 965-974.

[3] Barkhuus, L. and Dey, A. Location-based services for mobile telephony: a study of users' privacy concerns. Proc Interact, 2003.

[4] Blackwell, C., Birnholtz, J., and Abbott, C. Seeing and being seen: Co-situation and impression formation using Grindr, a location-aware gay dating app. New Media \& Society, 2014.

[5] Brown, G., Maycock, B., and Burns, S. Your picture is your bait: Use and meaning of cyberspace among gay men. Journal of Sex Research 42(1), 2005, 63-73.

[6] Bumgarner, B. Mobilizing the Gay Bar. presented at the International Communication Association, 2013, pp. 1-23.

[7] Ellison, N. and boyd, D. Sociality through Social Network Sites. In W.H. Dutton, ed., The Oxford Handbook of Internet Studies. Oxford University Press, Oxford, 2013, $151-172$ 
[8] Ellison, N., Heino, R., and Gibbs, J. Managing Impressions Online: Self-Presentation Processes in the Online Dating Environment. Journal of Computer-Mediated Communication 11(2), 2006, 415-441.

[9] Ellison, N.B., Hancock, J.T., and Toma, C.L. Profile as promise: A framework for conceptualizing veracity in online dating self-presentations. New Media \& Society 14(1), 2012, $45-62$.

[10] Fanon, F. Black sin, white masks. Paladin, London, 1970.

[11] Gross, R. and Acquisti, A. Information Revelation and Privacy in Online Social Networks. presented at the WPES, 2005, 1-10.

[12] Gudelunas, D. Online Personal Ads. Journal of Homosexuality 49(1), 2005, 1-33.

[13] Han, C.-S. They Don't Want To Cruise Your Type: Gay Men of Color and the Racial Politics of Exclusion. Social Identities 13(1), 2007, 51-67.

[14] Hancock, J.T. and Toma, C.L. Putting Your Best Face Forward: The Accuracy of Online Dating Photographs. Journal of Communication 59, 2 (2009), 367-386.

[15] Hong, L. and Jain, A. Integrating Faces and Fingerprints for Personal Identification. IEEE Transactions on Pattern Analysis and Machine Intelligence 20(12), 1998, 1295-1307.

[16] Humphreys, L. Mobile social networks and urban public space. New Media \& Society 12(5), 2010.

[17] Jain, A., Hong, L., and Pankanti, S. Biometric Identification. Communications of the ACM 43(2), 2000, 9198 .

[18] Jones, R.H. 'You show me yours, I'll show you mine': the negotiation of shifts from textual to visual modes in computer-mediated interaction among gay men. Visual Communication 4(1), 2005, 69-92.

[19] Kapp, M. Grindr. vanityfair.com, 2011. http://www.vanityfair.com/culture/features/2011/05/grindr201105.

[20] Kimmel, M. Grindr, Facebook and the new image culture. Gay-SD.com, 2013. http://www.gay-sd.com/grindrfacebook-and-the-new-image-culture/.

[21] Lang, N. A hidden epidemic. Salon.com, 2014. http://www.salon.com/2014/03/02/a hidden_epidemic_eatin g_disorders_in_the_gay_community/.

[22] Lea, M. and Spears, R. Computer-mediated communication, de-individuation and group decisionmaking. International Journal of Man-Machine Studies 34(2), 1991, 283-301.

[23] Lea, M. and Spears, R. Love at First Byte? Building Personal Relationships Over Computer Networks. In J.T. Wood and S. Duck, eds., Under-studied Realtionships: Off the Beaten Track. Sage Publications, London, 1995.

[24] Licoppe, C. and Inada, Y. Geolocalized Technologies, Location-Aware Communities, and Personal Territories: The Mogi Case. Journal of Urban Technology 15(3), 2008, 5-24.

[25] Litt, E. Knock, Knock. Who's There? The Imagined Audience. Journal of Broadcasting \& Electronic Media 56(3), 2012, 330-345.

[26] Long, J.S. and Freese, J. Regression Models for Categorical Depedent Variables Using Stata. Stata Press, College Station, TX, 2006.

[27] Megvii Inc. Face. www.faceplusplus.com, 2014. http://www.faceplusplus.com.

[28] Mowlabocus, S. Gaydar culture: Gay men, technology and embodiment in the digital age. 2010.

[29] Peterson, M.A. Perception of Faces, Objects, and Scenes. Oxford University Press, London, 2003.

[30] Phillips, P.J. and O'Toole, A.J. Image and Vision Computing. IMAVIS 32(1), 2014, 74-85.

[31] Phillips, P.J., Jiang, F., Narvekar, A., Ayyad, J., and O'Toole, A.J. An other-race effect for face recognition algorithms. ACM Transactions on Applied Perception 8(2), 2011, 1-11.

[32] Rosenfeld, M.J. and Thomas, R.J. Searching for a Mate: The Rise of the Internet as a Social Intermediary. American Sociological Review 77(4), 2012, 523-547.

[33] Rowlson, A. Not just a preference. fabmagazine.com, 2011. http://www.fabmagazine.com/story/not-just-apreference.

[34] Spears, R., Lea, M., and Lee, S. De-individuation and group polarization in computer-mediated communication. British Journal of Social Psychology 29, 1990, 121-134.

[35] Tajfel, H. and Turner, J.C. The social identity theory of intergroup behavior. In S. Worchel and W.G. Austin, eds., Psychology of intergroup relations. Nelson-Hall, Chicago, 1986, 7-24.

[36] Turner, J.C., Hogg, M.A., Oakes, P.J., Reicher, S.D., and Wetherell, M.S. A Self-Categorization Theory. In Rediscovering the Social Group: A Self-Categorization Theory. Basil Blackwell, Oxford, UK, 1987, 42-67.

[37] Woo, J. Meet Grindr. Amazon Digital Services, 2013.

[38] World Health Organization. Global Databse on Body Mass Index. apps.who.int, 2014.

http://apps.who.int/bmi/index.jsp?introPage=intro_3.html.

[39] Zhao, L., Lu, Y., and Gupta, S. Disclosure Intention of Location-Related Information in Location-Based Social Network Services. International Journal of Electronic Commerce 16(4), 2012, 53-89. 